\title{
Metabolismo do lactato: uma revisão sobre a bioenergética e a fadiga muscular
}

\section{Lactate metabolism: a review on bioenergetics and muscle fatigue}

\author{
Rômulo Cássio de Moraes Bertuzzi 1 \\ Adriano Eduardo Lima Silva ${ }^{1,2}$ \\ César Cavinato Cal Abad ${ }^{1}$ \\ Flávio de Oliveira Pires ${ }^{1}$
}

1. Universidade de São Paulo. Escola de Educação Física e Esporte. Laboratório de Determinantes Energéticos do Desempenho Esportivo. São Paulo, SP. Brasil.

2. Universidade Federal de Alagoas. Centro de Educação. Grupo de pesquisa em Ciências do Esporte. Maceió, AL. Brasil.

Recebido em 10/04/08 Revisado em 13/08/08 Aprovado em 15/11/08
Resumo - O objetivo desse trabalho foi apresentar os principais eventos que envolvem o metabolismo do lactato muscular, de modo a favorecer a compreensão acerca da utilização das concentrações sangüíneas de lactato ([La]) na estimativa do metabolismo anaeróbio lático e a sua possível relação com a fadiga muscular aguda. Tradicionalmente, o aumento das [La] esteve associado à redução do oxigênio mitocondrial. Porém, outros estudos demonstraram que demais substâncias, tais como a epinefrina e o fosfato inorgânico, são capazes de elevarem as [La]. A associação entre o lactato e o processo de fadiga muscular esteve baseada no aumento da acidose celular. No entanto, investigações recentes demonstraram que a acidose é capaz de restabelecer parcialmente a contração de músculos isolados e estimulados eletricamente. Nesse sentido, talvez a utilização das [La] na estimativa da contribuição do metabolismo anaeróbio lático seja limitada, bem como a relação deste metabólito com a fadiga muscular possa ser casual.

Palavras-chave: Acidose celular; Ácido lático; Anaeróbio lático; Exercício.

Abstract - The objective of this study was to report the main events involved in muscle lactate metabolism in order to contribute to the understanding of the use of blood lactate concentration (BLC) for the estimation of anaerobic lactate metabolism and their relationship with acute muscle fatigue. Traditionally, an increase in BLC is associated with insufficient mitochondrial oxygen supply. However, recent studies have shown that increased BLC might be due to the action of other agents such as epinephrine and inorganic phosphate. Similarly, the relationship between BLC and acute muscle fatigue has been based on an increase in cellular acidosis. However, recent studies have shown that cellular acidosis is able to partially restore the force of isolated and electrically stimulated muscle fibers. These findings suggest that BLC should be used with caution for the estimation of anaerobic lactate metabolism and that the relationship between BLC and acute muscle fatigue is only a casual phenomenon.

Key words: Cellular acidosis; Lactic acid; Anerobic lactic metabolism; Exercise 


\section{INTRODUÇÃO}

A taxa de conversão da energia química para mecânica durante a contração muscular é considerada um dos principais eventos fisiológicos determinantes do desempenho esportivo. Em linhas gerais, assume-se que durante os esforços de curta duração e com alta intensidade, a molécula de adenosina trifosfato (ATP) é ressintetisada, predominantemente, pela degradação da fosfocreatina e do glicogênio muscular, com subseqüente formação de lactato'.

Diferentemente do metabolismo aeróbio, a estimativa do metabolismo anaeróbio lático (MAL) é de difícil realização, sobretudo pela limitação de se acessar os marcadores fisiológicos que melhor o represente. No passado, o lactato foi considerado exclusivamente o produto final da degradação parcial da glicose/glicogênio devido à redução do oxigênio mitocondrial ${ }^{2}$. Esse modelo teórico foi criado a partir dos resultados de estudos do início do século XVII que demonstraram que a hipóxia ou anóxia induziam a produção do ácido lático $(\mathrm{AL})^{3}$.

Conseqüentemente, durante muitos anos, alguns preparadores físicos e pesquisadores da área esportiva acreditaram que o ácido lático (AL) fosse o principal fator limitante do desempenho humano ${ }^{4}$. Além disso, as concentrações sangüíneas de lactato ([La] também são comumente empregadas no estabelecimento da participação do metabolismo anaeróbio lático (MAL) durante o exercício físico, sobretudo, em tarefas que se aproximaram do gesto esportivo como no karatế ${ }^{5}$.

Todavia, a partir da década de 1990, muitos achados contribuíram para a mudança parcial do paradigma do $\mathrm{AL}$, em especial, ao que se refere a sua relação com a acidose intramuscular ${ }^{6}$ e a sua capacidade de representar o MAL ${ }^{3}$. Parte desse avanço tem-se dado pelo aprimoramento das técnicas empregadas nas investigações científicas? ${ }^{7}$. Dessa forma, com base nos trabalhos indexados na base Pubmed, os objetivos desta revisão de literatura foram: 1) descrever os principais eventos da produção e da remoção do lactato muscular; 2) apresentar os mecanismos que estabelecem a relação entre o lactato e a fadiga muscular; 3) apresentar a forma de utilização das [La] na estimativa do metabolismo anaeróbio lático e as suas possíveis limitações.

\section{PRODUÇÃO E REMOÇÃO DO LACTATO NO MÚSCULO ESQUELÉTICO}

O AL foi descoberto pelo químico sueco Carl Wilhelm Schelle, em amostras de leite, em meados de $1780^{6}$. Posteriormente, Otto Meyerhof compartilhou o prêmio Nobel em Fisiologia e Medicina, em 1922, com Archibald V. Hill, devido à descoberta da produção do lactato durante a contração muscular. Em linhas gerais, acreditava-se que o glicogênio muscular era degradado até gerar o AL e, subsequentemente, em lactato, para ressintetizar a ATP que é utilizada nas pontes cruzadas de miosina-actina e no processo ativo das bombas iônicas (Figura 1) ${ }^{4}$.

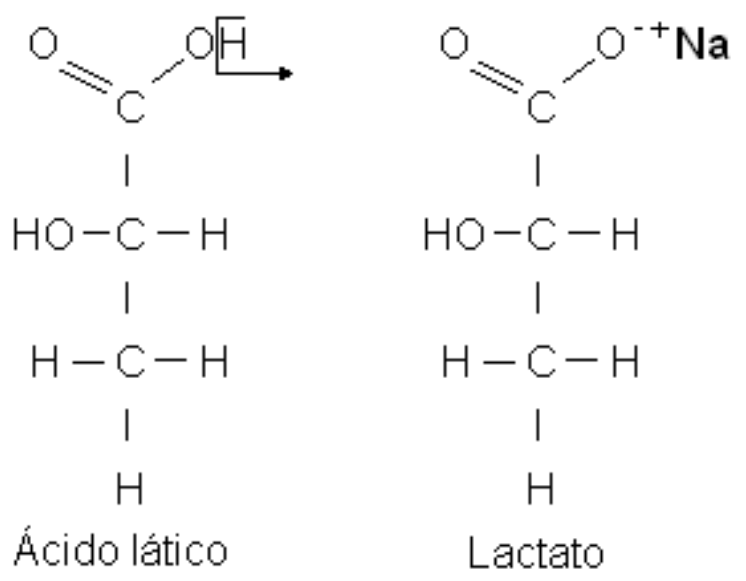

Figura 1. Estrutura química do ácido lático e do lactato. Quando o próton se dissocia do seu grupo funcional $\left(\mathrm{COOH}^{-}+\mathrm{H}^{+}\right)$ um cátion (nesse caso o sódio, $\mathrm{Na}^{+}$) interage com a negatividade do átomo de oxigênio do grupo carboxílico (adaptado de Robergs et al. ${ }^{7}$.

Pressupõe-se que a dissociação do AL no músculo esquelético acontece de forma relativamente rápida, pois a constante pKa dessa substância é de 3,87 em um ambiente com o $\mathrm{pH}$ entre 7,08 e 7,108. Assim, considerando-se que essa taxa de dissociação é dependente do equilíbrio ácido-base, substâncias que possuem a diferença de até mais ou menos uma unidade de $\mathrm{pH}$ mantêm a sua estrutura constante, ao passo que diferenças superiores ao valor da $\mathrm{pKa}$ tendem a facilitar a dissociação9. Essa diferença apresentada entre a constante $\mathrm{pKa}$ do $\mathrm{AL}$ e do $\mathrm{pH}$ do meio intramuscular resultará em aproximadamente $99 \%$ na dissociação dessa substância em prótons $\left(\mathrm{H}^{+}\right)$e ânions $\left(\mathrm{C}_{3} \mathrm{H}_{5} \mathrm{O}_{3}^{-}\right)$. Logo, durante a contração muscular intensa em humanos, as concentrações sarcoplasmáticas e plasmáticas de lactato podem chegar a 40 e $25 \mathrm{mmol} \cdot \lambda^{-1}$, respectivamente $^{4}$. A tabela 1 apresenta um breve resumo das principais propriedades desse ácido.

Por sua vez, a produção do lactato durante a atividade muscular foi considerada, por muito tempo, como um produto final da degradação parcial do glicogênio/glicose, em razão da baixa disponibilidade do oxigênio mitocondrial ${ }^{2}$. Entretanto, essa relação causal tem sido recentemente 
reformulada. Por exemplo, evidências mais recentes indicaram que ao induzir a redução de aproximadamente $22 \%$ do consumo de oxigênio $\left(\mathrm{WO}_{2}\right)$ das fibras musculares estimuladas eletricamente, as concentrações de lactato são mantidas semelhantes à situação controle ${ }^{10}$. Adicionalmente, Stainsby ${ }^{11}$ demonstrou que, em alguns estudos realizados com animais, foram observados aumentos da produção do AL sem a alteração da pressão de $\mathrm{O}_{2}$ no fluxo sangüíneo muscular.

Tabela 1. Resumo das propriedades químicas do ácido lático

\begin{tabular}{lc}
\hline Nome químico & $\begin{array}{c}\text { ácido 2-hidroxipropanoico } \\
\text { Fórmula química }\end{array}$ \\
Peso molecular $\left(\mathrm{g} \cdot \mathrm{mol}^{-1}\right)$ & 89,0 \\
Solubilidade & água, etanol, éter etíl \\
pKa $\left(37^{\circ} \mathrm{C}\right)$ & 3,87 \\
Calor de combustão & $321 \mathrm{kcal} \cdot \mathrm{mol}^{-1}$ \\
\hline
\end{tabular}

(Adaptado de Robergs et al. ${ }^{7}$ ).

Ao encontrar correlação significativa entre os pontos de inflexão das [La'] e de epinefrina, Brooks $^{12}$ reforçou a possibilidade da formação do AL independentemente da oferta de $\mathrm{O}_{2}$. Stainsby et al. ${ }^{13}$ corroboraram a hipótese de relação causal entre o aumento de epinefrina e o aumento da produção de lactato, ao observarem que o músculo gastrocnêmio de cães, estimulado eletricamente em conjunto com a infusão de epinefrina, elevava a produção de lactato em nível superior à situação controle. Neste caso, a ação da epinefrina deve estimular a quebra do glicogênio por meio da ativação da enzima glicogênio fosforilase ${ }^{13}$, transferindo um fosfato inorgânico à sua forma inativa. Como conseqüência da ação da epinefrina, haveria a ativação de toda a via glicolítica.

Outros fatores também podem interferir na produção do lactato, tais como o aumento da produção de insulina ${ }^{14}$, o conteúdo de glicogênio muscular e o consumo agudo de glicose ${ }^{15}$. Em linhas gerais, esses fatores seriam capazes de aumentar a disponibilidade do substrato energético para a via glicolítica. Por exemplo, a captação de glicose pelo músculo esquelético mediante a ação da insulina está positivamente correlacionada com aumento nas $\left[\mathrm{La}^{-}\right]^{14}$. Adicionalmente, Jacobs ${ }^{16}$ observou que os níveis musculares e sangüíneos de lactato são estatisticamente menores em relação à situação controle quando a depleção de glicogênio é induzida pela manipulação de dieta ou pelo exercício físico vigoroso. Porém, ele sugeriu que isso poderia ocorrer, possivelmente, em virtude do aumento da utilização do lactato durante o exercício e não necessariamente pela diminuição da sua produção.

De forma alternativa, tem-se proposto que pela interação de múltiplos eventos bioquímicos ${ }^{3}$ a mitocôndria não seria capaz de oxidar todos os piruvatos que são produzidos durante o esforço intenso, o que resultaria na sua conversão em lactado pela enzima lactato desidrogenase $(\mathrm{LDH})^{6}$. Isso, provavelmente, ocorre devido ao fato de algumas enzimas chaves do processo glicolítico, entre elas, a glicogênio fosforilase e a fosfofrutoquinase (PFK), terem os seus respectivos desempenhos alterados na presença de algumas substâncias químicas que atuam como sinalizadores. Por exemplo, a elevação das concentrações de Pi e de cálcio oriundas da contração muscular aumenta a atividade enzimática da glicogênio fosforilase (PHOS), ao passo que a sua inibição pode ser provocada pelo aumento de $\mathrm{H}^{+}$. Já a PFK aumenta sua atividade com a diminuição de ATP, bem como a elevação da amônia, epinefrina, AMP, ADP, Pi, pH e da frutose 1,6 difosfato ${ }^{2}$.

Por sua vez, a utilização do lactato como substrato energético pelo músculo esquelético ou por outros órgãos é possível graças ao seu transporte no meio intra e extracelular. Supõe-se que durante o exercício físico, sobretudo de intensidade elevada, o lactato produzido se desloque do meio intra para o extracelular mediante os transportadores monocarboxilatos (MCT1 e MCT4) ${ }^{17}$. Sugere-se que a isoforma MCT1 está presente em maior quantidade nas fibras de contração lenta, ao passo que a isoforma MCT4 está em maior quantidade nas fibras de contração rápida (Figura 2) ${ }^{14}$. Em relação as suas respectivas localizações, é provável que o MCT1 e o MCT4 estejam preferencialmente no sarcolema e na membrana da mitocôndria, respectivamente ${ }^{18}$. Thomas et al. ${ }^{17}$ detectaram transversalmente que a concentração de MCT1 era estatisticamente diferente entre grupos com diferentes níveis de aptidão aeróbia e que ela também estava positivamente correlacionada com a taxa de remoção das [La]. Além disso, já se demonstrou o aumento de aproximadamente $32 \%$ do MCT4 em sujeitos saudáveis após 6 semanas de treinamento resistido ${ }^{14}$, ao passo que nove semanas de treinamento aeróbio resultaram no acréscimo de, aproximadamente, $78 \%$ do MCT1 de indivíduos sedentários ${ }^{18}$.

Há evidências que demonstraram a presença da LDH e da MCT1 na mitocôndria ${ }^{18}$, fato que levaria a uma nova interpretação da relação entre os metabolismos anaeróbio lático e aeróbio, haja vista a possibilidade de transporte e da conversão do lactato em piruvato nessa organela. Em outras 
palavras, acredita-se que poderia haver a formação e acúmulo de piruvato dentro da mitocôndria mediante a ação da LDH. De forma similar, o fato de ser possível o transporte de piruvato para a mitocôndria por meio dos $\mathrm{MCT}^{3,18}$, reforça a necessidade de uma reformulação de parte dos conceitos aplicados à explicação da degradação incompleta dos carboidratos. Adicionalmente, a presença das isoformas MCT1 e MCT2 na fenda sináptica fortalece a proposição que o lactato também pode ser transportado entre os neurônios para ser utilizado como substrato energético ${ }^{3}$.

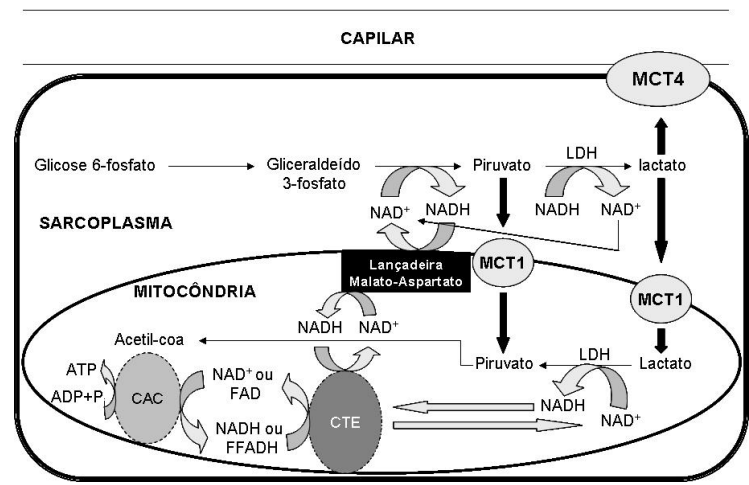

Figura 2. Ilustração das principais etapas do transporte e da degradação do lactato e do piruvato no meio intramuscular. Hipoteticamente, esses metabólitos poderiam ser transportados para a mitocôndria por meio dos transportadores monocarboxilatos (MCT). Em seguida, o lactato seria convertido em piruvato por meio da LDH dentro da mitocôndria (adaptado de Gladden $\left.^{3}\right)$. CTE = cadeia de transporte de elétrons; $\mathrm{CAC}=$ ciclo do ácido cítrico.

\section{LACTATO E FADIGA MUSCULAR AGUDA}

Tradicionalmente, a produção do lactato também esteve relacionada à liberação dos íons $\mathrm{H}^{+}$e à diminuição do $\mathrm{pH}$ intramuscular ${ }^{2}$, os quais seriam agentes depressores da contração ${ }^{19}$. As alterações no $\mathrm{pH}$ resultantes do acúmulo de $\mathrm{H}^{+}$teriam participação na inibição da liberação de $\mathrm{Ca}^{+}$, no aumento do $\mathrm{Ca}^{+}$livre, na inibição do motoneurônio, no tempo de transição do estado de ligação das pontes cruzadas de forte para fraca e na inibição das enzimas associadas à glicogenólise e à glicólise ${ }^{8}$. Portanto, $\mathrm{o} \mathrm{pH}$ intracelular diminui de forma progressiva com o aumento da duração do exercício intenso (Figura 3) seguido, supostamente, pela redução da capacidade de gerar tensão a partir do valor de $6,8^{4}$. Esse processo metabólico é denominado de acidose lática ${ }^{6}$.

Todavia, a associação entre o ácido lático, os íons $\mathrm{H}^{+}$e o desempenho físico tem sido questionada. Mais recentemente, Robergs et al. ${ }^{6}$ retomaram a discussão sobre as principais etapas do metabolismo energético que produzem os íons $\mathrm{H}^{+}$e, conseqüentemente, as causas da acidose celular e as limitações dos métodos que estimam o $\mathrm{pH}$ intracelular por meio da produção do lactato. Esses pesquisadores sugeriram que a glicólise isoladamente não seria capaz de produzir os prótons mencionados, haja vista que na reação intermediada pela fosfoglicerato quinase, a qual envolve a retirada de um fosfato inorgânico do 1,3-difosfoglicerato, formaria um dos ácidos carboxílicos (Tabela 2), os quais não possuem a capacidade de liberar prótons ${ }^{6}$. A seguir, as reações 1, 2 e 3 apresentam as principais etapas da liberação dos íons $\mathrm{H}^{+}$durante a contração muscular segundo Robergs et al. ${ }^{6}$.

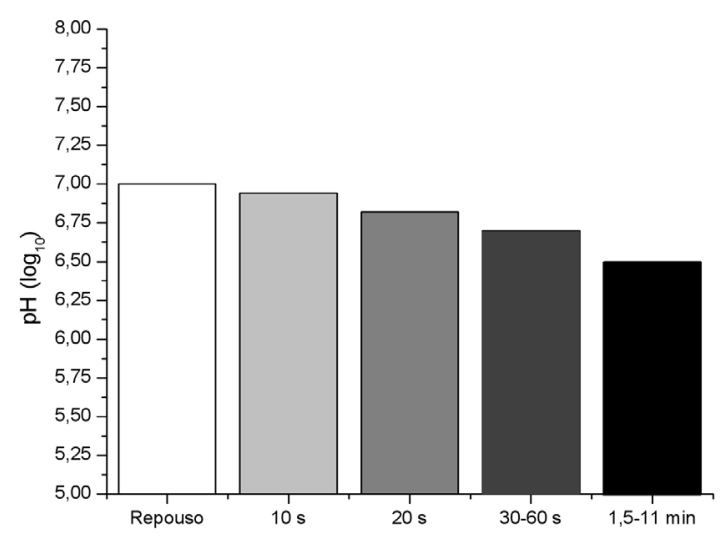

Figura 3. Valores médios esperados para o pH em função da duração do exercício físico intenso (adaptado de Cairns ${ }^{4}$ ).

Tabela 2. Valores da constante pKa dos demais ácidos intermediários da glicólise anaeróbia

\begin{tabular}{cc}
\hline 3-fosfoglicerato & 3,42 \\
2-fosfoglicerato & 3,42 \\
Fosfoenolpiruvato & 3,50 \\
Piruvato & 2,50 \\
\hline
\end{tabular}

(Adaptado de Robergs et al. ${ }^{6}$ ).

$$
\begin{aligned}
& \mathrm{ATP}+\mathrm{H}_{2} \mathrm{O} \stackrel{\text { ATPase }}{\longrightarrow} \mathrm{ADP}+\mathrm{Pi}+\mathbf{H}^{+} \text {REAÇÃO } 1 \\
& \text { Glicose + } 2 \mathrm{ADP}+2 \mathrm{Pi}+2 \mathrm{NAD}^{+} \\
& \rightarrow 2 \text { piruvatos }+2 \text { ATP }+2 \mathrm{NADH} \text { REAÇÃO } 2 \\
& +2 \mathrm{H}_{2} \mathrm{O}+2 \mathrm{H}^{+}
\end{aligned}
$$

$$
\text { Glicogênio + } 3 \text { ADP + 3Pi }+2 N A D^{+}
$$$$
\rightarrow 2 \text { piruvatos }+3 \mathrm{ATP}+2 \mathrm{NADH}
$$

REAÇÃO 3

$$
+2 \mathrm{H}_{2} \mathrm{O}+1 \mathbf{H}^{+}
$$

Robergs et al. ${ }^{6}$ também indicaram que a reação mediada pela $\mathrm{LDH}$ oxidaria a NAD para a formação do lactato, o que permitiria o fluxo do substrato para a segunda fase da glicólise. Assim, foi sugerido 
que, na verdade, a LDH atua como uma substância tampão e não acidificante do citoplasma, pois durante a formação do lactato via LDH há o consumo de $\mathrm{H}^{+}$(reação 4). Em outras palavras, tem-se proposto que, durante a degradação anaeróbia da glicose, não há formação de ácido lático ${ }^{21}$ e que a formação do lactato retardaria o desenvolvimento da acidose celular por agir como substância tampão ${ }^{6}$. Portanto, esses pesquisadores indicaram que a liberação dos íons $\mathrm{H}^{+}$durante a glicólise ou a glicogenólise ocorre de forma secundária, ou seja, somente pela hidrólise da ATP, em especial, nas reações que envolvem a hexoquinase e a PFK.

Piruvato $+\mathrm{NADH} \underset{\text { Lactato }+\mathrm{NAD}^{+}}{\stackrel{\text { Lactato }}{\text { desidrogenase }}}$

REAÇÃO 4

Além disso, Robergs et al. ${ }^{6}$ sugeriram que a acidose metabólica não ocorre somente pela liberação de próton, mas que ela é resultante do desequilíbrio entre a produção e o consumo dos íons $\mathrm{H}^{+}$. Esse contraponto foi fundamentado mediante os resultados de outros estudos experimentais que demonstraram que a quantidade de $\mathrm{H}^{+}$excede a produção de lactato durante a contração muscular. Assim, Robergs et al. ${ }^{6}$ dividiram o consumo total de prótons em três compartimentos, sendo estes: $1^{\circ}$ ) a idéia tradicional dos componentes que compõem o sistema de tamponamento intracelular estático ( $\mathrm{Pi}, \mathrm{HCO}_{3}$, IMP e aminoácidos); $2^{\circ}$ ) a utilização dos prótons durante a degradação da CP (reação 5); $3^{\circ}$ ) a utilização dos prótons durante a glicólise ou glicogenólise via LDH (reação 6). Por fim, Robergs et al. ${ }^{6}$ e Cairns ${ }^{4}$ indicaram que as evidências científicas que estabeleceram a relação causal entre a produção de lactato e a acidose celular foram baseadas apenas em observações correlacionais, as quais resultariam apenas na formulação de um constructo não factual.

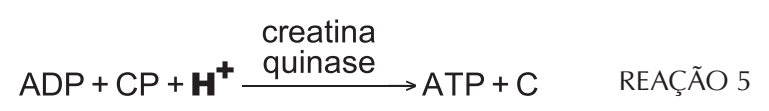

\section{2 fosfoenolpiruvato ${ }^{3-}$ \\ $+2 \mathrm{MgADP}^{-}+2 \mathbf{H}^{+}$ \\ piruvato quinase}

REAÇÃO 6

2 piruvatos +2 MgATP $^{2-}$

Por outro lado, Lindinger et al..$^{21}$ contra-argumentaram, afirmando que os conceitos apresentados por Robergs et al. ${ }^{6}$ e por Robergs e Parker ${ }^{20}$ foram baseados em outros constructos que também não representam a realidade dos fatos. Mais especificamente, Lindinger et al. ${ }^{21}$ criticaram as idéias propostas por Robergs et al. ${ }^{6}$ por não terem sido consideradas leis físicas que regem as reações químicas em meios aquosos: a conservação de massa e a manutenção da eletroneutralidade. Em linhas gerais, a eletroneutralidade é alcançada quando a diferença iônica no meio intracelular é igual à zero (reação 7). Segundo esses pesquisadores, o comportamento das moléculas é dependente das suas respectivas interações com a água, pois eles acreditam que devido à quantidade desse líquido ser muito maior que a quantidade de $\mathrm{H}^{+}$no músculo esquelético, ela providencia um suplemento infinito de prótons (reação 8) e que, de forma similar, os $\mathrm{H}^{+}$ também podem se reagrupar ao $\mathrm{HO}$. Nesse sentido, foi proposto que o aumento das concentrações dos ânions de lactato oriundos da glicólise induziria a dissociação dos íons $\mathrm{H}^{+}$contidos na água, no intuito de manter a eletroneutralidade. Em suma, Lindinger et al. ${ }^{21}$ alegaram que o lactato contribui diretamente para a acidose celular, haja vista que ele é um ânion que pode alterar o comportamento do citoplasma.

$$
\left(\left[\mathrm{Na}^{+}\right]+\left[\mathrm{H}^{+}\right]+\left[\mathrm{Ca}^{+}\right]+\left[\mathrm{K}^{+}\right]\right)-\left(\left[\mathrm{Cl}^{-}\right]+\left[\mathrm{La}^{-}\right]\right)=0
$$

REAÇÃO 7

$$
\mathrm{K}_{\mathrm{W}}\left[\mathrm{H}_{2} \mathrm{O}\right]=\left[\mathrm{H}^{+}\right]\left[\mathrm{HO}^{-}\right]
$$

REAÇÃO 8

Onde a constante de dissociação da água $\left(\mathrm{K}_{\mathrm{w}}\right)$ é muito pequena $\left(\cong 4,4 \times 10^{-14}(\mathrm{Eq} / \mathrm{l})^{2}\right)$ e as $\left[\mathrm{H}^{+}\right]$e $\left[\mathrm{HO}^{-}\right]$são muito baixas $\left(\cong 10^{-7} \mathrm{Eq} / \mathrm{l}\right)$.

$K_{e m p}{ }^{22}$ e Kemp et al. ${ }^{23}$ também refutaram parcialmente as proposições de Robergs et al. $^{6} \mathrm{e}$ Robergs e Parker ${ }^{20}$. Kemp ${ }^{22}$ indicou que, de fato, em repouso $(\mathrm{pH} \cong 7,0)$ os prótons seriam liberados principalmente pela hidrólise da ATP, mas à medida que o $\mathrm{pH}$ é reduzido durante a contração muscular $(\cong 6,4)$, a produção do lactato pela degradação do glicogênio assumiria o papel fundamental na liberação do $\mathrm{H}^{+}$. Kemp ${ }^{22}$ e Kemp et al. ${ }^{23}$ também afirmaram que para todos os valores de $\mathrm{pH}$, sempre há a liberação de um próton por lactato, independentemente do substrato utilizado. Adicionalmente, Kemp ${ }^{22}$ chamou atenção para o fato de que o consumo de prótons pela reação da creatina quinase é dependente da regulação metabólica, uma vez que a ativação da enzima glicogênio fosforilase interfere na disponibilidade de um dos seus substratos, o Pi. 
Contudo, cabe ressaltar que a maioria dos estudos supracitados que deram origem a essas hipóteses, não foi conduzida em temperaturas fisiológicas ${ }^{7,24}$. Logo, Westerblad ${ }^{7}$ sugeriram que se a redução do $\mathrm{pH}$ está envolvida na fadiga aguda, o efeito poderia ser indireto pela acidose extracelular, que ativaria os quimiorreceptores dos grupos III e IV das vias aferentes, os quais estariam relacionados à sensação de desconforto presente na fadiga aguda. Assim, alguma outra consequiência do metabolismo anaeróbio pode ser a causa verdadeira da queda da função muscular, como por exemplo, o aumento das concentrações do $P_{i}^{7}$. Essa elevação do $P_{i}$ resultante da hidrólise da ATP e da CP parece ter a capacidade de inibir a reabsorção do $\mathrm{Ca}^{+}$, que conseqüentemente influenciará no ambiente intracelular ${ }^{25}$.Contraditoriamente, outras investigações demonstraram que, além de não levar à fadiga, a acidose celular poderia ter um possível efeito protetor na manutenção da contração muscular. Por exemplo, após acidificarem os músculos gastrocnêmicos de ratos Wistar mediante o aumento de $\mathrm{CO}_{2}$ (de $5 \%$ para $24 \%$ ), Pedersen et al. ${ }^{26}$ constataram que a força muscular, previamente reduzida em $85 \%$, retornou $\mathrm{em} \cong 80 \%$ dos valores controle quando o $\mathrm{pH}$ foi reduzido de 7,4 para 6,8. Além disso, verificou-se também uma diminuição no trânsito de $\mathrm{Cl}$ extracelular ( $\cong 54 \%)$ após a acidificação. A partir desses resultados, a acidose parece ser capaz de recuperar a produção da força de músculos esqueléticos privados dessa função, possivelmente, pelo aumento da capacidade das fibras em iniciar e propagar os potenciais de ação, pela redução do $\mathrm{Cl}$ extracelular ou o bloqueio do seu canal na membrana.

De forma similar, Nielsen et al. ${ }^{24}$ verificaram que a capacidade de gerar tensão muscular foi completamente restabelecida após a acidose intramuscular mediante a infusão de $20 \mathrm{mM}$ de ácido lático em músculos gastrocnêmicos de ratos fatigados. Entretanto, nenhuma mudança significativa no potencial de repouso da membrana foi notada após a intervenção com o ácido lático. Adicionalmente, parte dos resultados do estudo de Paoli et al. ${ }^{27}$ demonstram que $75 \%$ da força é recuperada após o acréscimo de $10^{-5} \mathrm{M}$ de adrenalina em músculos que já haviam sido induzidos à fadiga pelo aumento de $15 \mathrm{mM}$ da concentração de $\mathrm{K}^{+}$extracelular e expostos à acidose pela infusão de $20 \mathrm{mM}$ de ácido lático $(\mathrm{pH}=6,8)$. Além disso, constatou-se que a infusão de epinefrina também resultou na hiperpolarização da membrana em razão da diminuição de $49 \%$ da concentração de $\mathrm{Na}^{+}$intracelular, mediante o aumento de $147 \%$ no funcionamento da bomba de $\mathrm{Na}^{+}-\mathrm{K}^{+}$. Com base nesses achados de Paoli et al. ${ }^{27}$ ,concluiu-se que: a) a acidose celular pode alterar a excitabilidade dos túbulos T no intuito de favorecer a despolarização das fibras; b) a epinefrina tem um efeito aditivo ao da acidose no restabelecimento da contração muscular, contudo a sua principal atuação está no restabelecimento do potencial de repouso da membrana.

Com base nesses resultados, é atraente suspeitar que a acidose lática pudesse ser benéfica no sentido de retardar a fadiga muscular, diferentemente da relação causal tradicional estabelecida. Dessa forma, o corpo editorial do Journal of Applied Physiology sistematizou um debate denominado Point:Counterpoint series: "Lactic acid accumulation is an advantage/disadvantage during muscle activity", no qual alguns especialistas foram convidados a apresentarem suas respectivas opiniões sobre o tema. Um resumo dessas informações está presente na Tabela 3. Parte das divergências deve-se às características das metodologias empregadas nos estudos realizados com músculos isolados e eletricamente estimulados. Bangsbo e Juel ${ }^{28}$ e Sahlin ${ }^{29}$ apontaram que os resultados dessas investigações são de difícil extrapolação para o exercício em humanos, pois: a) nesses trabalhos a estimulação elétrica não foi realizada de forma repetitiva e ela também não

Tabela 3. Pontos de vista acerca da influência da acidose lática na contração muscular.

\begin{tabular}{|c|c|}
\hline Pesquisadores & Ponto de vista \\
\hline $\begin{array}{l}\text { Lamb e Stephenson }^{30} \\
\text { Nielsen e Overgaard }\end{array}$ & $\begin{array}{l}\text { Acidose celular é benéfica para a contração muscular porque ela permite a propagação do } \\
\text { potencial de ação mediante o bloqueio dos canais de } \mathrm{Cl}^{-} \text {nos túbulos T. }\end{array}$ \\
\hline $\begin{array}{l}\text { Bangsbo e Juel }{ }^{28} \\
\text { Sahlin }{ }^{29}\end{array}$ & $\begin{array}{l}\text { Acidose celular é prejudicial à contração muscular porque ela contribui direta ou indiretamente } \\
\text { para a redução da capacidade do músculo gerar tensão, como por exemplo, pela liberação de } \\
\mathrm{K}^{+} \text {extracelular, pelo aumento do tempo no trânsito de } \mathrm{Ca}^{+} \text {e pela diminuição da afinidade do } \mathrm{O}_{2} \\
\text { com a hemoglobina. }\end{array}$ \\
\hline $\begin{array}{l}\text { Gladden e } \operatorname{Hogan}^{32} \\
\text { Lindinger } \\
\text { Burnley, Wilkerson e } \\
\text { Jones }^{34}\end{array}$ & $\begin{array}{l}\text { O efeito da acidose celular depende da intensidade do exercício. Nas intensidades até o } \\
\text { máximo estado estável de lactato ela é benéfica, pois ela permite a distribuição do substrato } \\
\text { energético e dos intermediários do metabolismo para os diferentes tecidos. Por outro lado, } \\
\text { durante o exercício intenso ela é prejudicial devido à sensação de desconforto e a dessaturação } \\
\text { da hemoglobina. }\end{array}$ \\
\hline
\end{tabular}


levou o músculo à exaustão; b) in vivo, a atividade da bomba de $\mathrm{N}^{+}-\mathrm{K}^{+}$é elevada tanto pelo aumento do $\mathrm{K}^{+}$extracelular como pela ação de hormônios, o que levaria ao restabelecimento do potencial de repouso da membrana e a manutenção da excitabilidade independentemente do $\mathrm{pH}$; c) a incubação dos músculos em ácido lático diminuiria menos o $\mathrm{pH}$ intracelular que o $\mathrm{pH}$ extracelular, criando assim a redução do gradiente do $\mathrm{pH}$ transmembranar, o qual é diferente do observado durante a contração muscular voluntária; d) quando os músculos são eletricamente estimulados é comum se observar falhas na contração, a qual é raramente detectada durante o exercício físico. Dessa forma, parece que é precoce afirmar que a acidose lática não está envolvida direta ou indiretamente com a fadiga muscular durante o exercício intenso.

\section{ESTIMATIVA DO METABOLISMO ANAERÓBIO LÁTICO POR MEIO DO LACTATO SANGÜÍNEO}

Embora se saiba que o fluxo glicolítico possui comportamento caótico a partir de uma determinada concentração de ATP $(\cong 1,2 \mathrm{mM})$ e de $\mathrm{NADH}(\cong$ 0,3 $\mathrm{mM})^{35}$, assume-se que, durante os esforços de alta intensidade ${ }^{36}$ ou quando a energia proporcionada pelo sistema oxidativo não é suficiente para atender a uma determinada demanda metabólica, a glicólise desempenha um papel proporcional ao da potência requerida nessas tarefas. Nesse sentido, as [La] têm sido comumente utilizadas para se estimar a capacidade do metabolismo aeróbio em testes ergométricos e a contribuição do sistema glicolítico no exercício físico ${ }^{36}$.

Os valores das [La] parecem ser sensíveis ao estado de treinamento dos indivíduos treinados anaerobiamente. Rehunen et al..$^{37}$ observaram que corredores velocistas possuíam valores superiores das [La] imediatamente após e no quinto minuto de recuperação de uma tarefa intermitente máxima, quando comparado com corredores de longa distância. $\mathrm{O}$ estudo de $\mathrm{Craig}^{38}$ demonstrou haver correlações significativas entre o pico da potência gerada em 30 e 40 segundos em cicloergômetro e o pico de acúmulo das [La] nessas tarefas.

Por sua vez, di Prampero e Ferretti ${ }^{36}$ afirmaram que é possível estimar o total de lactato produzido por quilo de massa corporal e que, através desse cálculo, haveria possibilidade de se adquirir informações sobre a energia transferida pelo metabolismo glicolítico. Neste caso, é necessário assumir que: as elevações das [La] sobre os níveis de repouso indu- zidos pelo exercício físico são acumuladas proporcionalmente no organismo, o pico de concentração sangüínea de lactato após o exercício é resultante de uma condição de equilíbrio entre o meio intracelular e extracelular, e que as taxas de remoção do lactato nos vários compartimentos de fluídos corporais têm a mesma constante de tempo.

Para tanto, assume-se que em exercício supramáximo e cíclico com duração superior a 30 segundos, a energia transferida por unidade de tempo (potência metabólica ou PM) seja representada pela somatória de dois termos: o primeiro refere-se ao metabolismo aeróbio e o segundo ao anaeróbio, podendo ser expresso pela equação $1^{36}$.

$\mathrm{PM}=\mathrm{PAM}+\beta\{\Delta(\mathrm{La})\}$

EQUAÇÃO 1

Onde $\mathrm{PM}=$ potência metabólica; $\mathrm{PAM}=$ potência aeróbia máxima (normalmente representada pelo $\left.\dot{\mathrm{V}} \mathrm{O}_{2 \max }\right) ; \beta=$ a constante gerada para representar a energia equivalente ao acúmulo de lactato; $\Delta[\mathrm{La}]=$ a taxa de produção de lactato, medida pela diferença entre o lactato de pico no sangue e o valor de lactato no repouso.

Embora não seja obtido o valor estequiométrico exato da oxidação do lactato a partir dessa equação, sugeriu-se que a constante $\beta$ proporciona dados satisfatórios para a estimativa da contribuição do metabolismo anaeróbio lático. Essa constante foi apresentada por di Prampero e Ferretti ${ }^{36}$ a partir do cálculo da inclinação de uma reta gerada com base na mensuração das [La] de pico para uma potência requerida em uma determinada tarefa. Quando os resultados são apresentados de forma relativa a PAM na corrida, natação ou no ciclismo, as retas geradas têm inclinações semelhantes, indicando que a energia proveniente do sistema glicolítico para os três exercícios são as mesmas $(\beta=3,0 \mathrm{ml}$ de $\mathrm{O}_{2} \cdot \mathrm{kg}^{-1} \cdot \mathrm{mM}^{-1} ; 2,7 \mathrm{ml}$ de $\mathrm{O}_{2} \cdot \mathrm{kg}^{-1} \cdot \mathrm{mM}^{-1}$ e $2,8 \mathrm{ml}$ de $\mathrm{O}_{2} \cdot \mathrm{kg}^{-1} \cdot \mathrm{mM}^{-1}$ para corrida, natação e ciclismo, respectivamente). Esses resultados levaram di Prampero e Ferretti ${ }^{36}$ a sugerirem a compatibilidade do valor fixo da constante $\beta$ de $3 \mathrm{ml} \mathrm{de} \mathrm{O}_{2} \cdot \mathrm{kg}^{1} \cdot \mathrm{mM}^{-1}$ para cada delta de $1 \mathrm{mM}$ de lactato de pico acima dos valores de repouso, viabilizando assim o cálculo da energia oriunda do sistema glicolítico por meio das [La] de pico.

Contudo, Gaesser e Brooks ${ }^{39}$ afirmaram que tanto em animais como em humanos a maior parte do lactato produzido durante e após o exercício é removido pela sua oxidação no músculo que o produziu. Brooks ${ }^{12}$ acredita ainda que, em seres humanos, esse comportamento parece ser linear em 
intensidades moderadas de esforço. Supostamente, o tipo de fibra que constitui um determinado grupo muscular poderia influenciar na remoção do lactato, pois as fibras de contração lenta teriam maior capacidade de oxidá-lo do que as de contração rápida ${ }^{3,12}$. De fato, Granier et al..$^{40}$ confirmaram que os grupos musculares com grande percentual de fibras lentas (antebraço com $\cong 50 \%$ ) tinham grande participação na remoção do lactato. Utilizando uma atividade intermitente supramáxima realizada com membros inferiores (cinco períodos de seis segundos de estímulo por cinco minutos de recuperação), esses pesquisadores verificaram que o lactato na amostra de sangue arterial do antebraço era significantemente maior após cada estímulo, quando comparado ao venoso.

Assim, apesar da estimativa do metabolismo anaeróbio utilizando as concentrações de lactato no sangue ser uma proposta atraente devido à facilidade de mensuração, a remoção do lactato pelos diferentes tecidos corporais, provavelmente em taxas diferentes em cada um deles, sugere cautela na utilização dessa metodologia.

\section{CONSIDERAÇÕES FINAIS}

Independentemente dos fatores que resultam na sua elevação, as [La'] têm sido amplamente utilizadas para se estimar a contribuição do metabolismo glicolítico durante o exercício físico. Contudo, essas inferências devem ser analisadas com cautela, pois o aumento das [La] não se dá apenas pela diminuição da oferta do oxigênio mitocondrial. De forma similar, ao longo dos anos, a associação entre o lactato e o processo de fadiga muscular aguda esteve baseada direta ou indiretamente ao aumento a acidose celular. Estudos mais recentes demonstraram que ela não é capaz de suprimir o funcionamento do aparato contrátil, indicando que a relação entre o aumento das [La] e a fadiga pode ser casual. Todavia, cabe ressaltar que as condições experimentais em que os músculos esqueléticos foram estimulados nesses estudos não se assemelharam fisiologicamente ao exercício físico voluntário.

\section{REFERÊNCIAS BIBLIOGRÁFICAS}

1. Medbo JI, Tabata I. Anaerobic energy release in working muscle during $30 \mathrm{~s}$ to $3 \mathrm{~min}$ of exhausting bicycling. J Appl Physiol 1993;75(4):1654-1660.

2. Brooks GA. Current concepts in lactate exchange. Med Sci Sports Exerc 1991;23(8):895-906.

3. Gladden LB. Lactate metabolism: a new paradigm for the third millennium. J Physiol 2004;1(558)(Pt 1):5-30.
4. Cairns SP. Lactic acid and exercise performance: culprit or friend? Sports Med 2006; 36(4):279-291.

5. Beneke R, Beyer T, Jachner C, Erasmus J, Hütler M. Energetics of karate kumite. Eur J Appl Physiol 2004; 92(4-5):518-523.

6. Robergs RA, Ghiasvand F, Parker D. Biochemistry of exercise-induced metabolic acidosis. Am J Physiol Regul Integr Comp Physiol 2004;287(3):R502-516.

7. Westerblad H, Allen DE, Lännergren J. Muscle fatigue: lactic acid or inorganic phosphate the major cause? News Physiol Sci 2002,17:17-21.

8. Fitts RH. Cellular mechanisms of fatigue muscle. Physiol Rev 1994;74(1):49-94.

9. Marzzoco A, Torres BB. Bioquímica básica. Guanabara Koogan, 1990.

10. Stainsby WN, Brooks GA. Control of lactic acid metabolism in contracting muscle and during exercise. Exerc Sport Sci Rev 1990;18:29-63.

11. Stainsby WN. Biochemical and physiological bases for lactate production. Med Sci Sports Exerc 1986;18(3):341-343.

12. Brooks GA. The lactate shuttle during exercise and recovery. Med Sci Sports Exerc 1986;18(3):360-368.

13. Stainsby WN, Brechue WF, O’Drobinak DM. Regulation of muscle lactate production. Med Sci Sports Exerc 1991;23(8):907-911.

14. Juel C, Holten MK, Dela F. Effects of strength training on muscle lactate release and MCT1 and MCT4 content in healthy and type 2 diabetic humans. J Physiol 2004; 1(n.556)(Pt 1):297-304.

15. Jacobs I. Blood lactate: implications for training and sports performance. Sports Exerc 1986;3:10-25.

16. Jacobs I. Lactate concentrations after short, maximal exercise at various glycogen levels. Acta Physiol Scand 1981;111(4):465-469.

17. Thomas C, Perrey S, Lambert K, Hugon G, Mornet D, Mercier J. Monocarboxylate transporters, blood lactate removal after supramaximal exercise, and fatigue indexes in humans. J Appl Physiol 2005;98(3):804-809.

18. Dubouchaud H, Butterfield GE, Wolfel EE, Bergman BC, Brooks GA. Endurance training, expression, and physiology of LDH, MCT1, and MCT4 in human skeletal muscle. Am J Physio End Metabolism 2000;278(4):E571-579.

19. Bergh U, Ekblom B, Astrand PO. Maximal oxygen uptake "classical" versus "contemporary" viewpoints. Med Sci Sports Exerc 2000;32(1):85-88.

20. Robergs RA, Parker D. Lingering construct of lactic acidosis. Am J Physiol Regul Integr Comp Physiol 2005;289:R904-910.

21. Lindinger MI, Kowalchuk JM, Heigenhauser GJ. Applying physicochemical principles to skeletal muscle acid-base status. Am J Physiol Regul Integr Comp Physiol 2005; 289(3):R891-894.

22. Kemp G. Lactate accumulation, proton buffering, and $\mathrm{pH}$ change in ischemically exercising muscle. Am J Physiol Regul Integr Comp Physiol 2005;289:R895-901.

23. Kemp G, Böning D, Beneke R, Maassen N. Explaining $\mathrm{pH}$ change in exercising muscle: lactic acid, proton consumption, and buffering vs. strong ion differen- 
ce. Am J Physiol Regul Integr Comp Physiol 2005 291:R235-237.

24. Nielsen OB, de Paoli FV, Overgaard K. Protective effects of lactic acid on force production in rat skeletal muscle. J Physiol 2001;1(536)(Pt 1):161-166.

25. Favero TG. Sarcoplasmic reticulum $\mathrm{Ca}^{+}$release and muscle fatigue. J Appl Physiol 1999; 87(2):471-483.

26. Pedersen TH, de Paoli F, Nielsen OB. Increased excitability of acidified skeletal muscle: role of chloride conductance. J Gen Physiol 2005;125(2):237-246.

27. de Paoli FV, Overgaard K, Pedersen TH, Nielsen OB. Additive protective effects of the addition of lactic acid and adrenaline on excitability and force in isolated rat skeletal muscle depressed by elevated extracellular $\mathrm{K}+$. J Physiol 2007;581(Pt 2):829-839.

28. Bangsbo J, Juel C. Point:couterpoint: Lactic acid accumulation is an advantage/disadvantage during muscle activity. J Appl Physiol 2006;100(4):1412-1413.

29. Sahlin K. Point:couterpoint: Lactic acid accumulation is an advantage/disadvantage during muscle activity. J Appl Physiol 2006;101(1):367.

30. Lamb GD, Stephenson DG. Point:couterpoint: Lactic acid accumulation is an advantage/disadvantage during muscle activity. J Appl Physiol. 2006;100(4):1410-1412.

31. Nielsen OB, Overgaard K. Point:couterpoint: Lactic acid accumulation is an advantage/disadvantage during muscle activity. J Appl Physiol 2006;101(1):367.

32. Gladden LB, Hogan MC. Point:couterpoint: Lactic acid accumulation is an advantage/disadvantage during muscle activity. J Appl Physiol 2006;100(6):2100-2101.

33. Lindinger MI. Point:couterpoint: Lactic acid accumulation is an advantage/disadvantage during muscle activity. J Appl Physiol 2006;100(6):2100.

34. Burnley M, Wilkerson DP, Jones AM. Point:couterpoint: Lactic acid accumulation is an advantage/disadvantage during muscle activity. J Appl Physiol 2006;101(2):683.
35. Nielsen K, Sorensen PG, Hynne F. Chaos in Glycolysis. J Theor Biology 1997; 186(3):303-306.

36. di Prampero PE, Ferretti G. The energetics of anaerobic muscle metabolism: a reappraisal of older and recent concepts. Respir Physiol 1999;1(118):10-15.

37. Rehunen S, Näveri H, Kuoppasalmi K., Härkönen M. High-energy phosphate compounds during exercise in human slow-twitch and fast-twitch muscle fibers. Scand J Clin Lab Invest 1982;42(6):499-506.

38. Craig NP, Pyke FS, Norton KI. Specificity of test duration when assessing the anaerobic lactacid capacity of high-performance track cyclists. Int J Sports Med 1989;10(4):237-242.

39. Gaesser AG, Brooks GA. Metabolic bases of excess post-exercise oxygen consumption: a review. Med Sci Sports Exerc 1984;16(1):29-43.

40. Granier P, Dubouchaud H, Mercier B, Mercier J, Ahmaidi S, Préfaut CH. Lactate uptake by forearm skeletal muscles during repeated periods of short-term intense leg exercise in humans. Eur J Appl Physiol 1996;72(3):209-214.

\section{Agradecimento}

Bolsa de demanda social CAPES.

\section{Endereço para correspondência}

Rômulo Cássio de Moraes Bertuzzi

Rua Clorindo de Oliveira Cajé, 91.

CEP: 05371-140 - Butantã, São Paulo. SP. Brasil

E-mail: bertuzzi@usp.br 